\title{
SARS-CoV-2 seroprevalence in children and their family members, July-October 2020, Brussels
}

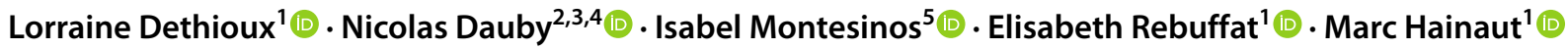

Received: 19 April 2021 / Revised: 24 August 2021 / Accepted: 3 October 2021 / Published online: 22 October 2021

() The Author(s), under exclusive licence to Springer-Verlag GmbH Germany, part of Springer Nature 2021

\begin{abstract}
The aim of this study was to estimate the seroprevalence of SARS-CoV-2 antibodies in a pediatric population after the first pandemic wave in Belgium. All patients requiring a blood sample between 1 July 2020 and 31 October 2020 in our institution were invited to participate. Their parents and siblings could also participate to estimate familial transmission and the congruence between serological statuses. A questionnaire was completed for each participant to identify symptoms consistent with COVID-19 in the previous months. Blood samples were tested for SARS-CoV-2-specific immunoglobulin G using ELISA. The final population included 112 children, 24 siblings of these children, and 36 adults. The seroprevalence of cases was $6.9 \%$ before 8 September, a date that corresponds to 1 week after the beginning of the second wave in Belgium and $22.5 \%$ afterwards ( $\mathrm{OR}=3.89,95 \% \mathrm{CI}(1.20 ; 12.58)$, $p$-value $=0.03$ ). Twenty-five percent of children were asymptomatic, and none experienced severe disease. The symptoms associated with SARS-CoV-2-positive antibodies were diarrhoea $(\mathrm{OR}=9.9,95 \% \mathrm{CI}[2.88 ; 33.87 .65] p$-value $<0.01)$, fever $(\mathrm{OR}=3.8,95 \%$ CI $[1.44 ; 10.22] p$-value $<0.01)$, rhinitis $(\mathrm{OR}=3.9,95 \%$ CI $[1.38 ; 10.90] p$-value $=0.01)$, or anosmia $(\mathrm{OR}=31.5,95 \% \mathrm{CI}[1.45$; 682.7], $p$-value $=0.02$ ). A child was the first symptomatic household member in $50 \%$ of the familial clusters.

Conclusion: Seroprevalence in children was comparable to that of the general population. Children could represent the source of infection in the household.
\end{abstract}

\section{What is Known:}

- COVID-19 infection is generally mild or asymptomatic in children and adolescents.

- Belgian strategy of testing was focused on symptoms.

- Adults are believed to be responsible for most of familial clusters.

What is New:

- Serological testing gives a more accurate view of the rate of infected children.

- Based on serological results, children have been infected as frequently as adults during the first and second wave in Belgium.

- Seventy-five percent of SARS-CoV-2 IgG-positive children presented a mild symptomatology, and $25 \%$ were totally asymptomatic.

- Children could represent the source of infection within household.

Keywords Children $\cdot$ SARS-CoV-2 antibodies $\cdot$ Seroprevalence $\cdot$ Familial transmission

Communicated by Nicole Ritz

Lorraine Dethioux

lorraine.dethioux@ulb.be

Nicolas Dauby

nicolas.dauby@stpierre-bru.be

Isabel Montesinos

mariaisabel.montesinoshernandez@lhub-ulb.be

Elisabeth Rebuffat

elisabeth.rebuffat@stpierre-bru.be

Marc Hainaut

marc.hainaut@stpierre-bru.be
1 Pediatrics Department, CHU Saint-Pierre, Université Libre de Bruxelles (ULB), Brussels, Belgium

2 Infectious Diseases Department, CHU Saint-Pierre, Université Libre de Bruxelles (ULB), Brussels, Belgium

3 School of Public Health, Université Libre de Bruxelles (ULB), Brussels, Belgium

4 Institute for Medical Immunology, Université Libre de Bruxelles (ULB), Brussels, Belgium

5 Microbiology Department, Laboratoire Hospitalier Universitaire de Bruxelles (LHUB-ULB), Brussels, Belgium 


\section{Abbreviations \\ $\mathrm{CHU}$ \\ Centre Hospitalier Universitaire \\ COVID-19 \\ Coronavirus disease 2019 \\ $\mathrm{IgG}$ \\ PCR \\ Immunoglobulin $\mathrm{G}$}

SARS-CoV-2 Severe acute respiratory syndrome coronavirus 2

\section{Introduction}

Severe acute respiratory syndrome coronavirus 2 (SARSCoV-2), the virus that causes coronavirus disease 2019 (COVID-19), has spread rapidly around the world since emerging in Wuhan, China, in late 2019 [1]. In Belgium, the first case of the new respiratory disease was detected in February 2020, and local transmission of SARS-CoV-2 infections was subsequently proven in early March. There was a first wave of infections from March to the end of June 2020, then a low incidence of new COVID-19 infections until end of August, and a second wave started after August 31 [2].

The clinical course of infected individuals is highly variable, ranging from asymptomatic carriage to very severe disease, eventually leading to death. The potential for transmission is documented at all stages, with pre-symptomatic and asymptomatic transmissions playing an important role in the pandemic [3].

The usual diagnostic method of COVID-19 is the detection of SARS-CoV-2 by polymerase chain reaction (PCR) on a nasopharyngeal swab. As elsewhere, the testing strategy has gradually evolved in Belgium. At the beginning of the first wave, only those requiring hospitalisation were screened, whereas since May 2020, it is recommended to test every possible case.

Even if their burden varies widely among countries, children and adolescents are consistently a low proportion among people diagnosed with COVID-19 [4]. However, the pediatric population is likely underreported in epidemiologic studies as testing is focused on symptoms, and children and young people have generally a mild clinical course of the disease [4]. Specific antibodies testing could therefore be a more accurate method to estimate the real impact of SARS$\mathrm{CoV}-2$ in children as they reveal previous infections regardless of the symptoms. The cumulative incidence estimated by SARS-CoV-2 antibody detection was already demonstrated to be much higher than that ascertained by reported COVID-19 cases [5].

The results of first seroprevalence studies tend to confirm that children have lower rates of infection than adults, especially the younger children ( $<10$ years) [6-8], but this is not uniformly found, and some studies show the same seroprevalence in children as in adults [9-11].

The role of children in the epidemic, particularly in household transmission, remains also poorly understood. However, several studies show that the secondary attack rate is lower if the index case is a child $[12,13]$.

The objective of this study was to estimate the seroprevalence of SARS-CoV-2 antibodies in a pediatric population after the first pandemic wave in Belgium and to get insight into the route of contamination within household clusters.

\section{Materials and methods}

\section{Study design}

This monocentre observational study was designed to estimate the seroprevalence of SARS-CoV-2 antibodies among children requiring a blood sample for any reason (follow-up, pre-operative, diagnostic assessment...) between 1 July 2020 and 31 October 2020 in different settings from the Centre Hospitalier Universitaire (CHU) Saint-Pierre (Brussels). Their parents and siblings were also invited to participate to estimate familial transmission and the congruence between serological statuses. Parents were asked to complete a questionnaire to identify whether they or their child had developed symptoms consistent with COVID-19 at any time since the beginning of the pandemic.

\section{Participants}

Children aged between 6 months and 16 years at the time of recruitment who needed a blood sample for any reason were eligible to participate. Informed consent was obtained, and assent was given by children 9 years or older. If the informed consent was given for the child, we offered parents living with the child and any siblings to be included as well. Participants were free to decline/withdraw consent at any time without providing a reason and without being subject to any resulting detriment.

\section{Serological testing}

Blood samples were collected at the CHU Saint-Pierre, and the sera were tested for SARS-CoV-2-specific immunoglobulin $\mathrm{G}(\mathrm{IgG})$ using the Euroimmun Anti-SARS-CoV-2 ELISA IgG (Euroimmun, Luebeck, Germany) according to the manufacturer's instructions for the enzyme-linked immunosorbent assay (ELISA) automated system ETI-MAX 3000 (DiaSorin, Saluggia, Italy). The Euroimmun Anti-SARS-CoV-2 ELISA IgG uses microplate wells that are coated with the SARS$\mathrm{CoV}-2$ recombinant $\mathrm{S} 1$ structural protein, and the results are evaluated semi-quantitatively by calculation of a ratio of the extinction of samples over the extinction of the calibrator. This test demonstrated excellent sensitivity and specificity 
for detection of $\mathrm{IgG}$ antibodies from samples collected after COVID-19 diagnosis by polymerase chain reaction (PCR) [14]. The ratio interpretation was as follows: $<0.8$ : negative, $\geq 0.8$ to $<1.1$ borderline, $\geq 1.1=$ positive.

Each enrolment included a questionnaire on age, sex, medical background, contact with PCR-positive individuals, place of work, school, and kindergarten frequentation. Symptoms compatible with COVID-19 since March 2020 and up to 15 days before the serological test were also recorded. Each participant was contacted by phone to be informed of their results and eventually complete any missing information. A retrospective review of medical files was also made when necessary.

\section{Statistical analysis}

Descriptive statistics were used for demographic data and symptomatology. Variables associated with SARS-CoV-2 infection were analysed with Mann-Whitney $U$ tests for continuous data and Fisher's exact test for categorical data. All the analyses were carried out using GraphPad QuickCalcs (GraphPad Software, San Diego, CA, USA). A $p$-value of 0.05 or less was considered statistically significant.

\section{Results}

We included 115 children as well as 24 siblings and 37 parents ( 15 fathers and 22 mothers) of these children. Recruitment of children by site is divided as follows: emergency wards (46\%), dermatology and ear, nose and throat outpatient clinic (36\%), pediatric hospitalisation wards (11\%), and pre-operative outpatient consultations (7\%).

The participants whose SARS-CoV-2 ELISA test result was determined as borderline were excluded from the analysis. The final population therefore included 112 children, 24 siblings of these children, and 36 adults.

\section{Seroprevalence in children}

We focused the analysis of the seroprevalence of SARSCoV-2 infections after the first wave in Belgium on the children who were initially recruited (one child per household) to avoid the bias of selecting children from households in which the virus had been more prevalent, which could have been the case if several children from the same family had been included. The median age of the 112 children was 4.5 years (range 0.5 to 15.4 years), 60 (53.6\%) were boys. The presence of anti-SARS-CoV-2 IgG was demonstrated in 14 children (12.5\%) with a proportion of seropositive children significantly higher after the 8th of September, a date that corresponds to one week after the beginning of the second wave in Belgium. There were 5/72 (6.9\%) SARS-CoV-2-positive sera among those sampled between 1 July and 8 September vs 9/40 (22.5\%) among those sampled after this date $(p$-value $=0.03, \mathrm{OR}=3.89$, $95 \%$ CI $[1.20 ; 12.58])$. The median age of the SARSCoV-2 seropositive children was 9.2 years, compared to 4 years for SARS-CoV-2 seronegative children. However, the age difference was not statistically significant $(p=0.1)$. Parents' professional activities during lockdown, school attendance during lockdown, housing conditions, medical history were not associated with past SARS-CoV-2 infection. Only a notion of a contact with a PCR-positive individual was significantly associated with SARS-CoV-2 seropositivity (3/98 vs $7 / 14, p$-value $<0.0001, \mathrm{OR}=31.67$, 95\% CI $[6.68 ; 150.0]$ ) (Table 1$)$.

\section{Familial testing}

We offered to perform serologic testing on siblings and parents of all 112 children, but only 31 households agreed to participate. The members of these households were distributed as follows: 31 initially recruited children, 24 siblings, 15 fathers, and 21 mothers.
Table 1 Characteristics of patients with a positive SARSCoV-2 IgG. Based only on initially recruited patients $(n=112)$

\begin{tabular}{lllll}
\hline & $\begin{array}{l}\text { Total } \\
n=112\end{array}$ & $\begin{array}{l}\text { SARS-CoV-2 IgG } \\
\text { seronegative } n=98\end{array}$ & $\begin{array}{l}\text { SARS-CoV-2 } \\
\text { IgG seropositive } \\
n=14\end{array}$ & $P$ Value \\
\hline Molecular testing & 26 & 23 & 3 & $P>0.05$ \\
Male & 60 & 52 & 8 & $P>0.05$ \\
House living & 22 & 18 & 4 & $P>0.05$ \\
Unemployed parents & 34 & 30 & 4 & $P>0.05$ \\
Professional activity during lockdown* & $34 / 78$ & $31 / 68$ & $3 / 10$ & $P>0.05$ \\
School attendance during lockdown** & $11 / 66$ & $10 / 60$ & $1 / 6$ & $P>0.05$ \\
Identified risk contact & 10 & 3 & 7 & $P<0.01$ \\
Relevant health history & 35 & 31 & 4 & $P>0.05$ \\
\hline
\end{tabular}

*Seventy-eight children had at least one parent with a professional activity at the time of lockdown. The rest of the parents was either unemployed or absent

${ }^{* *}$ Sixty-six children were attending school at the time of lockdown 
Six households (19.4\%) had at least one seropositive member (Table 2). These 6 households comprised 21 analysable subjects, 7 were adults, and 14 children (aged 1.5 to 16.8 years). The first symptomatic case was a child in 3 households and an adult in 3 other families.

In these households, the majority of adults (6/7) and children (13/14) reported mild symptoms compatible with COVID-19 at any moment from March 2020. None required hospitalization. However, the detection of SARS-CoV-2 antibodies was more frequent in children than in adults as we detected SARS-CoV-2 IgG in $2 / 7$ adults and in $12 / 14$ children ( $p$-value $=0.02, \mathrm{OR}=15.0$, $95 \%$ CI $[1.63 ; 138.2])$.

Interestingly, among the 3 children who had a negative PCR at the time of the symptoms, 2 exhibited SARSCoV-2-specific IgG (Table 2).

\section{Symptoms in children}

Including the 24 siblings, 136 children were tested for the presence of SARS-CoV-2 antibodies. Among them, 20 were SARS-CoV-2 IgG seropositive (14 initially recruited children and 6 siblings; median age 8.3 years, range from 0.6 to 15.4 years).

Of the 20 seropositive children, 15 (75\%) experienced viral infection symptoms. No symptoms were noted by their parents for 5 . The prevalence of asymptomatic infection was therefore $25 \%$. Overall, 49 children experienced symptoms which could have been consistent with COVID-19 infection, but only 15 exhibited SARS-CoV-2 antibodies (30.6\% of symptomatic children).

Among the 136 children, the report of any symptom of a viral infection since March 2020, but also specifically the report of an episode of diarrhoea $(p$-value $<0.01, \mathrm{OR}=9.87,95 \%$ CI $[2.88 ; 33.87])$, fever ( $p$-value $<0.01, \mathrm{OR}=3.84,95 \% \mathrm{CI}[1.44 ; 10.22])$, rhinitis $(p$-value $=0.01, \mathrm{OR}=3.88,95 \%$ CI $[1.38 ; 10.90])$, or anosmia $(p$-value $=0.02, \mathrm{OR}=31.49,95 \%$ CI $[1.45 ; 682.7])$ were all significantly associated with the presence of specific SARS-CoV-2 IgG (Table 3). By contrast, the report of a sore throat, myalgia, dyspnoea, or chest pain were not associated with the serology result. No children were hospitalised because of SARS-CoV-2 infection in our study.

\section{Discussion}

In Belgium, in order to reduce social contacts and thus the circulation of the virus in the population, a general lockdown has been imposed in mid-March 2020, and in particular, schools have been closed with the exception of daycare centres for children whose parents work in an "essential"

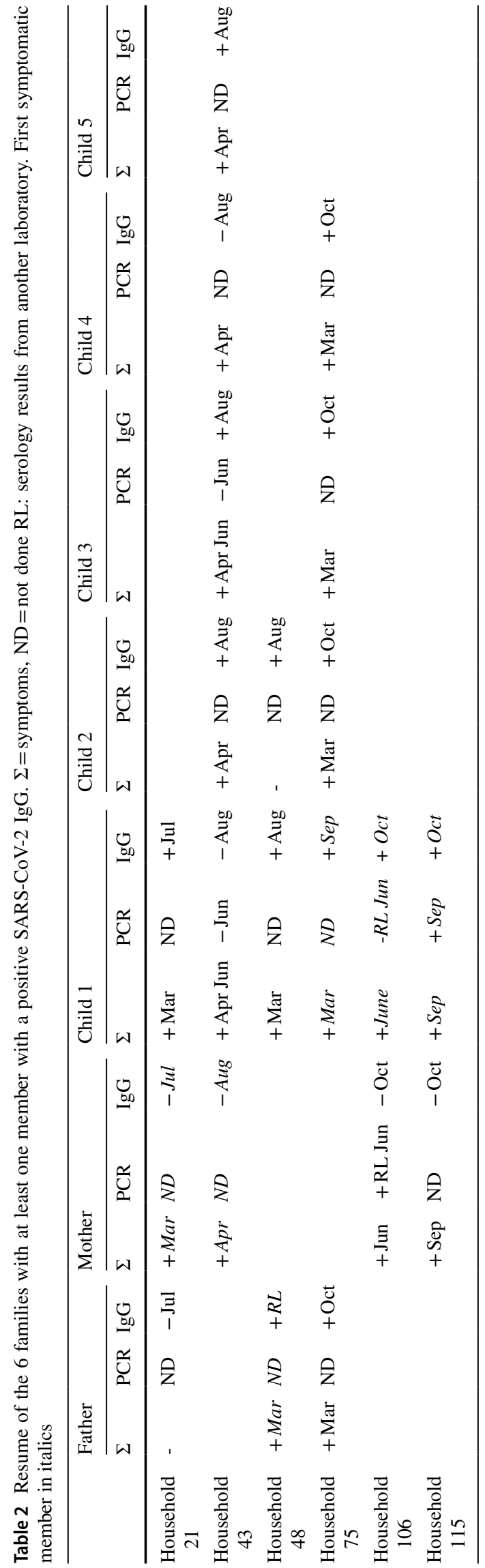


Table 3 Symptomatology according to serological status. Based on all children: initially recruited patients + siblings $(n=136)$

\begin{tabular}{llllr}
\hline Symptoms & Total & $\begin{array}{l}\text { Sars-CoV-2 IgG seronegative } \\
\text { patients: } n=116(\%)\end{array}$ & $\begin{array}{l}\text { Sars-CoV-2 IgG seropositive } \\
\text { patients: } n=20(\%)\end{array}$ & $P$-Value \\
\hline None & 89 & $84(72)$ & $5(25)$ & $P<0.01$ \\
Fever & 39 & $28(24)$ & $11(55)$ & $P<0.05$ \\
Rhinitis & 25 & $17(15)$ & $8(40)$ & $P<0.05$ \\
Cough & 23 & $16(14)$ & $7(35)$ & $P<0.05$ \\
Diarrhoea & 13 & $6(5)$ & $7(35)$ & $P<0.01$ \\
Sore throat & 7 & $4(3)$ & $3(15)$ & $P>0.05$ \\
Myalgia & 7 & $4(3)$ & $3(15)$ & $P>0.05$ \\
Dyspnoea & 3 & $3(3)$ & $0(0)$ & $P>0.05$ \\
Anosmia & 2 & $0(0)$ & $2(10)$ & $P<0.01$ \\
Chest pain & 0 & $0(0)$ & $0(0)$ & $P>0.05$ \\
Total & 136 & $116(100)$ & $20(100)$ & \\
\hline
\end{tabular}

profession. A progressive release of the lockdown was then carried out from mid-May. The seroprevalence we found in children after the first wave $(6.9 \%)$ is in the range of what was found in the Belgian population at the same time period (4.5 to $6.9 \%$ ) [15]. We show also a significantly higher SARS-CoV-2 seroprevalence $(22.5 \%)$ after September 8, which corresponds to 1 week after the beginning of the second wave in Belgium. However, mitigation measures were only reintroduced at the end of October. In the meantime, the children could engage in all their usual activities with the only constraint of having to wear a mask for those over the age of 12. Compared to the first wave, during which schools were closed and people were confined to their homes, the potential sources of infection were much greater for children at the beginning of the second wave, increasing their likelihood of being infected. The increase in seroprevalence from the beginning of the second wave is in line with what was seen in the adult population for this period (26\% in Brussels) [16]. The seroprevalence we found is relatively comparable to those found at the same time in the adult population in Belgium, both after the first wave and at the beginning of the second wave.

Meta-analysis of household contact studies has shown fewer cases in children compared to adults [17], although these findings may be subject to bias [18]. However, children have significantly more potentially contaminating contacts than adults when no mitigation measures are taken, and this could explain that we, as others, found serological evidence that the rate of infection in children is comparable to that of the adult population [9]. In our study, SARS-CoV-2-seropositive children tended to be older than seronegative children. The difference was not statistically significant potentially due to the small sample size. Younger children might have reduced exposure due to a lower degree of social mobility or may be less likely to seroconvert [19].

Transmission within households, particularly depending on the age of the index case, is still subject to many uncertainties. Current evidence supports a lower transmission from children to adults [1, 20, 21], but data are not unequivocal as one study found younger age $(<20$ years) associated with increased infectivity [22] and other studies finding no age effect [23, 24]. However, few studies have characterized the transmission of SARS-CoV-2 in European households and the risk for transmission depends on virological factors but also on the type and intensity of social contacts, which may differ between different cultures.

Many studies on household transmission were done when a strict confinement was applied and children had few or no social interactions outside of the family. In our study, as many children as adults seem to have brought the virus into their home and most of the children living in the households where we have the serologic proofs that the virus was introduced were immunised against SARS-CoV-2. Therefore, it seems that, even if children show few signs of infections, they could be easily infected when they are placed in high-risk conditions to acquire the SARS-CoV-2. The transmission by children in their households could also be more frequent than initially thought. A high transmission of SARS-CoV-2 among US household members, including households where the index case was a child, was recently described [25], and transmission from children or adolescents to other family members showed a secondary attack rate of $45 \%$ in one study [26]. Recently, a Canadian study showed that, among households in which the apparent primary case was a child, the youngest children are the most likely to spread the virus, with the highest odds of transmission observed for children aged 0 to 3 years [24].

Two of the three children who were PCR negative at the time of symptoms had SARS-CoV-2-specific IgG afterwards. One study in children suggests that they could mount a cellular immune response to SARS-CoV-2 more easily than adults and sometimes without virological confirmation of infection, raising the possibility that immunity in children can prevent the establishment of SARS-CoV-2 infection 
[27]. A prospective household contact study suggests that infected children are detectable by PCR for a shorter period than adults [28].

Most of the children who exhibited SARS-CoV-2 IgG in our study were never tested by a molecular method, although 75\% did develop symptoms of a viral infection, either because their minor symptoms did not require medical consultation or because they did not meet the national testing guidelines criteria. As only cases proven by molecular diagnosis are recorded in the national database, this could lead to a large bias in the pediatric figures if they are less often symptomatic or less symptomatic than adults.

We found that at least $25 \%$ of SARS-CoV-2-infected children never developed symptoms. The retrospective design of our study with respect to symptoms raises the possibility that the symptoms reported by parents may not be related to SARS-CoV-2 and that the number of children who were actually asymptomatic at the time of their infection is actually higher. Given the intense focus on health in Belgium since the first wave of COVID, it is unlikely that parents have forgotten symptoms presented by their child. The prevalence of asymptomatic SARS-CoV-2 infections we found is roughly the same as what is described in adults [29] and is in the range of asymptomatic infections numbers in other pediatric studies [30, 31].

By comparing the symptoms presented by positive SARS-CoV-2 IgG children with negative SARS-CoV-2 children, we found four symptoms associated with seropositivity: fever, diarrhoea, rhinitis, and anosmia. These symptoms were previously reported in other studies involving children [32-34] but cannot be seen as specific for COVID-19 and are frequently very self-limited and mild in children. It is not obvious whether there are more asymptomatic infections in children than in adults but more infections remain mild and do not lead to a medical contact [35].

This study has several limitations. The retrospective design is prone to recall bias, and minor symptoms might not have been identified. It is possible that parents and children were more willing to participate if they suspected they had been infected, introducing a possible selection bias. We were also only able to test 6 households with at least 1 seropositive member. With such low numbers, errors due to chance (by sampling fluctuations) are more frequent. Finally, its monocentric design gives us information localised to a single geographical area located in downtown Brussels. The findings might have been different in area with distinct socio-demographic and economic characteristics [36].

However, we offer important epidemiological data on the rate of SARS-CoV-2 infection in Belgian children. These data provide more objective information on the impact of the pandemic on the pediatric population than those obtained from the nasopharyngeal swab, which underestimates the infection rate in children. The findings that the SARS-CoV-2 seroprevalence in children is comparable to adults, suggesting that they are as frequently infected as adults but that the infection stays under-recognised in children are of importance, as are the findings that children could not unusually be the first case in households.

In conclusion, we do not find a lower seroprevalence of infection in children compared to adults. On the contrary, this seroprevalence seems to be at least as high, both by comparing our data with adult Belgian data from the same period and by the number of people confirmed to be infected in the households in which there was demonstrated SARSCoV-2 infection. Children have mostly minor symptoms when infected and are therefore under-tested and underdiagnosed. However, it is not such a rare event that a child is the primary source of infection in a household.

Acknowledgements This research would not have been possible without support from: "Lions Clubs International DISTRICT 112C" under the leadership of Ghislaine Estiévenart (governor 2019/2020) and the support of Dr Véronique Moreau. We would like to thank all the pediatric department, the sampling centre, and the LHUB for their deep involvement in this research. We also thank our colleagues Chadia Lfaqir, Dr Marie-Paule Thill, Dr Athanassios Kolivras, Dr Frederic Mergan, Dorothy Cauchie, and Evelyne Van Der Kelen for their help in the present work.

Authors' contributions L. Dethioux, N. Dauby, and M.Hainaut contributed to the design and implementation of the research, to the analysis of the results, and to the writing of the manuscript. I. Montesinos supervised the blood samples analysis and the corresponding part of the manuscript. E. Rebuffat supervised the project.

Funding Sponsored by Lions Clubs International DISTRICT $112 \mathrm{C}$ under the leadership of Ghislaine Estiévenart Governor 2019/2020. N.D. is a clinical master post-doctorate of the F.R.S-FNRS.

Data vailability (data transparency) N/A

Code availability (software application or custom code) N/A

\section{Declarations}

Ethics approval This study was performed in line with the principles of the Declaration of Helsinki and was approved by the local ethical committee under the CE/20-06-15 registration number.

Informed consent Informed consent was obtained for each participant, and assent was given by children 9 years or older.

Consent to participate and for publication Informed consent was obtained for each participant, and assent was given by children 9 years or older.

Disclaimer There is no financial relationship between the authors and the funder. The funder had no role in the design and conduct of the study; collection, management, analysis, and interpretation of the data; preparation, review, or approval of the manuscript; and decision to submit the manuscript for publication. 
Conflict of interest The authors declare no competing interests.

\section{References}

1. Dattner I, Goldberg Y, Katriel G et al (2020) The role of children in the spread of COVID-19: using household data from Bnei Brak, Israel, to estimate the relative susceptibility and infectivity of children. medRxiv 2020.06.03.20121145. https://doi.org/10. $1101 / 2020.06 .03 .20121145$

2. Sciensano determination de la date marquant le debut de la deuxième vague de covid-19 en belgique. https://covid-19. sciensano. be/sites/default/files/Covid19/Second\%20Wave_FR.pdf

3. Buitrago-Garcia D, Egli-Gany D, Counotte MJ et al (2020) Occurrence and transmission potential of asymptomatic and presymptomatic SARS-CoV-2 infections: a living systematic review and meta-analysis. PLoS Med 17:e1003346. https://doi.org/10.1371/ journal.pmed.1003346

4. Idele P, Anthony D, You D et al (2020) The evolving picture of SARS-CoV-2 and COVID-19 in children: critical knowledge gaps. BMJ Glob Health 5:e003454. https://doi.org/10.1136/ bmjgh-2020-003454

5. Havers FP, Reed C, Lim T et al (2020) Seroprevalence of antibodies to SARS-CoV-2 in six sites in the United States, March 23-May 3, 2020. medRxiv 2020.06.25.20140384. https://doi.org/ 10.1101/2020.06.25.20140384

6. Stringhini S, Wisniak A, Piumatti G et al (2020) Seroprevalence of anti-SARS-CoV-2 IgG antibodies in Geneva, Switzerland (SEROCoV-POP): a population-based study. The Lancet 396:313-319. https://doi.org/10.1016/S0140-6736(20)31304-0

7. Pollán M, Pérez-Gómez B, Pastor-Barriuso R et al (2020) Prevalence of SARS-CoV-2 in Spain (ENE-COVID): a nationwide, population-based seroepidemiological study. The Lancet 396:535-544. https://doi.org/10.1016/S0140-6736(20)31483-5

8. Pagani G, Conti F, Giacomelli A et al (2020) Seroprevalence of SARS-CoV-2 significantly varies with age: preliminary results from a mass population screening. J Infect 81:e10-e12. https:// doi.org/10.1016/j.jinf.2020.09.021

9. Brotons P, Launes C, Buetas E et al (2021) Susceptibility to severe acute respiratory syndrome coronavirus 2 infection among children and adults: a seroprevalence study of family households in the Barcelona Metropolitan Region, Spain. Clin Infect Dis 72:e970-e977. https://doi.org/10.1093/cid/ciaa1721

10. Hobbs CV, Drobeniuc J, Kittle T et al (2021) Estimated SARS-CoV-2 seroprevalence among persons aged $<18$ years - Mississippi, MaySeptember 2020. MMWR Morb Mortal Wkly Rep 70:312-315. https://doi.org/10.15585/mmwr.mm7009a4

11. Bi Q, Wu Y, Mei S et al (2020) Epidemiology and transmission of COVID-19 in 391 cases and 1286 of their close contacts in Shenzhen, China: a retrospective cohort study. Lancet Infect Dis 20:911-919. https://doi.org/10.1016/S1473-3099(20)30287-5

12. Madewell ZJ, Yang Y, Longini IM et al (2020) Household transmission of SARS-CoV-2: a systematic review and meta-analysis of secondary attack rate. medRxiv 2020.07.29.20164590. https:// doi.org/10.1101/2020.07.29.20164590

13. Kim J, Choe YJ, Lee J et al (2021) Role of children in household transmission of COVID-19. Arch Dis Child 106:709-711. https://doi.org/10.1136/archdischild-2020-319910

14. Montesinos I, Gruson D, Kabamba B et al (2020) Evaluation of two automated and three rapid lateral flow immunoassays for the detection of anti-SARS-CoV-2 antibodies. J Clin Virol 128:104413. https://doi.org/10.1016/j.jcv.2020.104413
15. Herzog S, Bie JD, Abrams S et al (2020) Seroprevalence of IgG antibodies against SARS coronavirus 2 in Belgium - a serial prospective cross-sectional nationwide study of residual samples. medRxiv 2020.06.08.20125179. https://doi.org/10.1101/ 2020.06.08.20125179

16. Augmentation du nombre de donneurs de sang et de travailleurs de la santé ayant developpé des anticorps contre le coronavirus. In: sciensano.be. https://www.sciensano.be/fr/coin-presse/augmentation-dunombre-de-donneurs-de-sang-et-de-travailleurs-de-la-sante-ayantdeveloppe-des. Accessed 16 Jan 2021

17. Viner RM, Mytton OT, Bonell C et al (2021) Susceptibility to SARS-CoV-2 infection among children and adolescents compared with adults: a systematic review and meta-analysis. JAMA Pediatr 175:143-156. https://doi.org/10.1001/jamapediatrics. 2020.4573

18. Hyde Z (2021) Difference in severe acute respiratory syndrome coronavirus 2 attack rate between children and adults may reflect bias. Clin Infect Dis. https://doi.org/10.1093/cid/ciab183

19. Irfan O, Li J, Tang K et al (2021) Risk of infection and transmission of SARS-CoV-2 among children and adolescents in households, communities and educational settings: a systematic review and meta-analysis. J Glob Health 11:05013. https://doi.org/10. 7189/jogh.11.05013

20. Lee B, Raszka WV (2020) COVID-19 transmission and children: the child is not to blame. Pediatrics e2020004879. https://doi.org/ 10.1542/peds.2020-004879

21. Merckx J, A. Labrecque J, S. Kaufman J, (2020) Transmission of SARS-CoV-2 by children. Dtsch Arztebl Int 117:553-560. https:// doi.org/10.3238/arztebl.2020.0553

22. Li F, Li Y-Y, Liu M-J et al (2021) Household transmission of SARS-CoV-2 and risk factors for susceptibility and infectivity in Wuhan: a retrospective observational study. Lancet Infect Dis 21:617-628. https://doi.org/10.1016/S1473-3099(20)30981-6

23. Sun K, Wang W, Gao L et al (2021) Transmission heterogeneities, kinetics, and controllability of SARS-CoV-2. Science 371:eabe2424. https://doi.org/10.1126/science.abe2424

24. Paul LA, Daneman N, Schwartz KL et al (2021) Association of age and pediatric household transmission of SARS-CoV-2 infection. JAMA Pediatr. https://doi.org/10.1001/jamapediatrics.2021.2770

25. Grijalva CG, Rolfes MA, Zhu Y et al (2020) Transmission of SARS-COV-2 infections in households - Tennessee and Wisconsin, April-September 2020. MMWR Morb Mortal Wkly Rep 69:1631-1634. https://doi.org/10.15585/mmwr.mm6944e1

26. Chu VT, Yousaf AR, Chang K et al (2021) Household transmission of SARS-CoV-2 from children and adolescents. N Engl J Med. https://doi.org/10.1056/NEJMc2031915

27. Tosif S, Neeland MR, Sutton P et al (2020) Immune responses to SARS-CoV-2 in three children of parents with symptomatic COVID-19. Nat Commun 11:5703. https://doi.org/10.1038/ s41467-020-19545-8

28. Lewis NM, Duca LM, Marcenac P et al (2021) Characteristics and timing of initial virus shedding in severe acute respiratory syndrome coronavirus 2, Utah, USA. Emerg Infect Dis 27:352-359. https://doi.org/10.3201/eid2702.203517

29. Yanes-Lane M, Winters N, Fregonese F et al (2020) Proportion of asymptomatic infection among COVID-19 positive persons and their transmission potential: a systematic review and meta-analysis. PLoS ONE 15:e0241536. https://doi.org/10.1371/journal.pone.0241536

30. Poline J, Gaschignard J, Leblanc C et al (2020) Systematic SARS$\mathrm{CoV}-2$ screening at hospital admission in children: a French prospective multicenter study. Clin Infect Dis. https://doi.org/10. 1093/cid/ciaa1044

31. Allan-Blitz L-T, Hertlein F, Klausner JD (2021) Prevalence of asymptomatic severe acute respiratory syndrome coronavirus 2 
infection among youth. Pediatr Infect Dis J 40:e132. https://doi. org/10.1097/INF.0000000000003023

32. Mehta NS, Mytton OT, Mullins EWS et al (2020) SARS-CoV-2 (COVID-19): what do we know about children? A systematic review. Clin Infect Dis 71:2469-2479. https://doi.org/10.1093/ $\mathrm{cid} / \mathrm{ciaa} 556$

33. Waterfield T, Watson C, Moore R et al (2020) Seroprevalence of SARSCoV-2 antibodies in children: a prospective multicentre cohort study. Arch Dis Child. https://doi.org/10.1136/archdischild-2020-320558

34. Mak PQ, Chung K-S, Wong JS-C et al (2020) Anosmia and ageusia: not an uncommon presentation of COVID-19 infection in children and adolescents. Pediatr Infect Dis J 39:e199. https:// doi.org/10.1097/INF.0000000000002718
35. Davies NG, Klepac P, Liu Y et al (2020) Age-dependent effects in the transmission and control of COVID-19 epidemics. Nat Med 26:1205-1211. https://doi.org/10.1038/s41591-020-0962-9

36. Burström B, Tao W (2020) Social determinants of health and inequalities in COVID-19. Eur J Public Health 30:617-618. https:// doi.org/10.1093/eurpub/ckaa095

Publisher's Note Springer Nature remains neutral with regard to jurisdictional claims in published maps and institutional affiliations. 